\title{
IGUALDAD BÁSICA Y PERSONA MORAL
}

\author{
Olof Page \\ Instituto de Filosofía, Pontificia Universidad Católica de Chile
}

\begin{abstract}
Resumen
El objetivo del artículo es analizar y criticar los argumentos elaborados por Carlos S. Nino para justificar una cierta interpretación del concepto de persona moral. Esta interpretación ofrece una estrategia de fundamentación de los derechos morales básicos distinta a la contenida en lo que en el artículo llamo "la estrategia tradicional". El cambio radical de estrategia que Nino propone implica una cierta concepción del igual (o desigual) estatus moral de los seres humanos. Por las razones dadas en el artículo, creo que este cambio de estrategia no es exitoso.
\end{abstract}

PALABRAS CLAVE: Igualdad básica; Persona moral; Propiedades.

\begin{abstract}
The objective of the article is to analyze and criticize the arguments elaborated by Carlos S. Nino to justify a certain interpretation of the concept of moral person. This interpretation offers a foundation of basic moral rights that is different from the one contained in what in the article I call "the traditional strategy". The radical change of strategy that Nino proposes implies a certain conception of the equal (or unequal) status of human beings. For the reasons given in the article, I think that this change of strategy is unsuccessful.
\end{abstract}

KEY WORDS: Basic Equality; Moral Persons; Properties.

La idea de que los seres humanos son moralmente iguales entre sí parece ser una creencia ampliamente difundida, al punto que se podría decir que un criterio o principio moral es considerado como válido en la medida en que supone la verdad de esta creencia. La misma noción de discriminación arbitraria, así como es usada en la práctica común, es moral y políticamente relevante en la medida en que se supone que los seres humanos son moralmente iguales. No quiero decir con esto que la noción de discriminación arbitraria necesariamente deba suponer la igualdad moral para ser operativa. Nada impide que tal discriminación se dé entre seres que no tienen igual estatus moral. Si existe la creencia 
compartida de que ciertos seres humanos tienen un valor moral inferior al valor de otros seres humanos, existiría discriminación arbitraria si se establece la existencia de, por ejemplo, iguales derechos fundamentales pues, si las personas no son moralmente iguales, no deberían tener iguales derechos fundamentales (excluyendo razones de corte instrumental o pragmático que pudiesen justificar una igual distribución). Por supuesto, el que alguien afirme que algunos seres humanos valen más que otros nos suele parecer moralmente repugnante y, por esta razón, el tipo de discriminación recién mencionada no se constituye, en nuestro contexto cultural, en una práctica. Esto muestra que la creencia en la igualdad moral opera en los hechos como un supuesto al que suele dársele el estatus de obviedad, es decir, un supuesto que, en caso de ser necesario, podría ser respaldado por buenas razones. Este hecho se ve confirmado por la práctica filosófica misma, que no ha lidiado críticamente con esta creencia de manera proporcional a la importancia, casi axiomática, que se le atribuye. Estoy de acuerdo con Jeremy Waldron cuando, en relación con la discusión sobre la igualdad moral, afirma que "analíticamente, el estado de la discusión es una desgracia comparado con el de la literatura que se centra en [la discusión de la igualdad como un propósito económico o social]" (Waldron 2008, p. 1). Esto es lamentable, entre otras cosas, porque la pregunta "iigualdad de qué?" -entendida como una pregunta relativa a qué es lo que debería ser distribuido igualitariamente para que una determinada comunidad política se considere justa-no puede contar con una respuesta completa y debidamente justificada si no se responde primero la pregunta “¿por qué igualdad?", es decir, por qué eso que se considera importante distribuir (por ejemplo, derechos, recursos materiales, libertades, oportunidades, etc.) debe ser distribuido igualitariamente y, además, por qué se considera importante distribuir eso y no otra cosa. En relación a este último punto es razonable suponer una conexión relevante entre el bien seleccionado a distribuir y eso que se cree justifica la creencia en la igualdad moral; es decir, si afirmamos, por ejemplo, que es nuestra condición de agentes morales lo que justifica nuestra creencia en la igualdad moral, el bien (o bienes) a distribuir debería guardar alguna relación con esa condición. En general, la idea es que un determinado bien debe ser distribuido igualitariamente porque los seres humanos son, en algún sentido, iguales y que la razón por la que se los considera iguales es también uno de los motivos para distribuir igualitariamente ese bien y no otro ${ }^{1}$. Si los seres humanos no fueran

${ }^{1}$ Un tratamiento más detallado de la relación entre igualdad moral e igualdad distributiva se encuentra en Carter y Page (ms.). 
iguales, entonces no existirían razones no instrumentales para distribuir un determinado bien igualitariamente ${ }^{2}$. Por este motivo, examinar si existen razones que justifiquen la creencia en la igualdad moral entre los seres humanos es una cuestión importante, cuyos problemas centrales son, como veremos, de fácil formulación pero difíciles de tratar. En lo que sigue llamaré a este tipo de igualdad "igualdad básica". Por razones de simplicidad haré uso de este término en relación a los seres humanos, sin que esto implique negar ni aceptar la posibilidad de que la igualdad básica pueda predicarse también de un conjunto más amplio de seres.

En un estimulante ensayo escrito en inglés y publicado en 1987 en la revista mexicana Crítica, titulado "The Concept of Moral Person", Carlos Nino propone, según sus palabras, "un cambio radical de estrategia filosófica"3 (p. 140) para abordar la cuestión de la titularidad de los derechos fundamentales (o derechos humanos) y su relación de justificación con lo que aquí llamo igualdad básica. Esta estrategia representaría un cambio respecto de la siguiente posición: si los seres humanos son iguales, en virtud de que poseen ciertas propiedades igualmente, entonces tienen iguales derechos morales ${ }^{4}$. Si no fueran iguales porque no poseen ciertas propiedades igualmente, entonces no tendrían iguales derechos morales. Llamaré a esta posición "la estrategia tradicional", para oponerla a la estrategia de cambio radical que Nino propone. Mi intención en este trabajo es poner en cuestión la plausibilidad de este cambio radical de estrategia. Creo que hacer esto es relevante porque las observaciones críticas de Nino sobre la igualdad básica son de gran valor y, por esa razón, ponerlas en cuestión es una oportunidad para pensar algunas de las dificultades más agudas que encierra este importante tema. Desgraciadamente no tengo, por el momento, nada constructivo que proponer sobre este punto, de manera que mis observaciones críticas a los argumentos de Nino no deberían interpretarse como una defensa de la estrategia tradicional.

${ }^{2}$ En Cupit (2000) se encuentra un argumento para justificar la igual distribución de un determinado bien que no se basa en la igualdad entre seres humanos sino en la noción de individualidad. No examinaré aquí la plausibilidad de esta propuesta.

${ }^{3}$ Las citas corresponden a la versión en español de este artículo que aparece en Nino (2007).

${ }^{4}$ Nino habla aquí indistintamente de "grados en la extensión en que esos derechos son reconocidos" y de "gozar algunos derechos" dada la existencia de cierta propiedad relevante, como "el hecho de ser humano" (p. 138). Como veremos, la distinción entre ser titular de un derecho y gozar de él es relevante en la defensa del cambio radical de estrategia que Nino elabora. 
Primero mencionaré las razones que Nino ofrece para justificar este cambio de estrategia (secciones I-III) y luego señalaré por qué su estrategia me parece fallida (secciones III-IV). El argumento general de Nino es complejo y, por lo mismo, su exposición requiere un nivel de elaboración que tomará varias páginas. Sin embargo, si comparamos el nivel de la discusión en el que Nino se mueve con el que se da en la literatura reciente sobre el tema, los méritos de su ensayo resaltan todavía más y, por esta razón, el esfuerzo de clarificar las diferentes partes de este argumento vale la pena. ${ }^{5}$

\section{II}

Según Nino, cuando adoptamos el punto de vista de la estrategia tradicional no existen buenas razones que permitan asignarle un "sentido adecuado" (p. 138) a la frase "todos los seres humanos son iguales" pues no es posible identificar una propiedad (o conjunto de propiedades) que todos los miembros del conjunto relevante -en este caso los seres humanos- posean igualmente y que, en consecuencia, justifique la creencia en la existencia de iguales derechos morales.

Cuando, adoptando esta estrategia, intentamos interpretar la frase "Todos los seres humanos son iguales" tenemos, al menos, tres posibilidades. Según Nino, bajo ninguna de ellas la estrategia tradicional funcionaría.

a. La interpretación descriptiva, que sería falsa porque los seres humanos difieren en muchos de sus rasgos.

b. La interpretación normativa, que sería falsa pues "hasta el igualitarista más convencido admite que no todos los hombres deben ser tratados de igual manera" (p. 138).

c. La interpretación analítica que, en principio, parece aceptable: "ser humano", en la frase "Todos los seres humanos son iguales", tiene carácter de "todo o nada" y, en consecuencia, todos los seres humanos son igualmente seres humanos.

La interpretación analítica nos permitiría justificar nuestra creencia en la igualdad básica pues la propiedad "ser humano" no admite grados, es decir, se es o no se es un ser humano y, por tanto, si $x$ es un ser humano y $w$ es un ser humano, $x$ y $w$ son igualmente seres humanos y de allí ciertas consecuencias normativas se siguen. Estas consecuencias

${ }^{5}$ Algunos de los trabajos recientes o relativamente recientes sobre igualdad básica son Williams (1962); Rawls (1971); Lloyd Thomas (1979); Arneson (1999); Cupit (2000); Waldron (2002) y (2008); Carter (2011). 
normativas serían de corte igualitario y se justifican mediante lo que Nino llama "la característica formal de la superveniencia de los principios morales", que afirma que

no es posible establecer soluciones normativas distintas entre casos, a menos que existan diferencias en propiedades fácticas relevantes [...]. En consecuencia, si la única propiedad relevante para gozar de algunos derechos es el hecho de ser un ser humano, y esta condición no admite consideraciones de grado, significa entonces que no pueden existir diferencias de grado en la extensión en que esos derechos son reconocidos, esto es, todos los seres humanos deben tenerlos en el mismo grado (p. 138).

Pero Nino se pregunta: “¿es cierto que el predicado "ser humano" no es de carácter gradual?" (p. 139). Su respuesta es negativa. Por esta razón, si basamos la distribución de derechos en esa propiedad, tal distribución debería ser desigual. La interpretación analítica, a pesar de su plausibilidad inicial, también es falsa. Examinemos las razones que justifican esta afirmación.

Nino sostiene que, ya que el predicado "ser humano" no es primitivo, como el predicado "rojo", si es o no gradual dependerá de las propiedades más primitivas que lo caracterizan. Cuando entendemos estas propiedades más primitivas en términos biológicos, el carácter no gradual parece asegurado pero surge otro problema. El criterio biológico -por ejemplo, tener células con 46 cromosomas en su núcleo- no sería moralmente relevante para determinar quién debe ser acreedor de derechos morales fundamentales. Si creyéramos que sí es relevante, tal creencia sería, según Nino, semejante a posiciones racistas que reconocen derechos solo a miembros de una determinada raza humana porque los miembros de esa raza comparten ciertas propiedades biológicas que justifican que ellos posean ciertos derechos que, quienes no son miembros de esa raza, no deben poseer. Aquí no habría racismo pero sí especismo (p. 139).

Es posible también analizar el predicado "ser humano" en términos de propiedades como la racionalidad, inteligencia o capacidad de decisión o de elegir valores. Estas propiedades tienen una ventaja respecto del criterio anterior pues estas sí parecen relevantes moralmente a la hora de adscribir derechos como la libertad de expresión o de religión (p. 139). Sin embargo, el problema es que estas propiedades son graduales, de manera que los seres humanos serían titulares de derechos en "diferente extensión, de acuerdo con su grado de racionalidad, 
inteligencia", etc. (p. 140). Esta mirada elitista es incluso más chocante [shocking] que la especista, afirma Nino (pp. 139-140).

Dados los problemas mencionados, estamos frente a un "dilema desagradable" (p. 140): o la propiedad escogida no es gradual pero es moralmente irrelevante, o es moralmente relevante pero gradual. Lo que debemos hacer es, según Nino, rechazar la presuposición que nos conduce a este dilema. La presuposición es la siguiente:

el concepto de persona moral debe denotar a una clase de individuos (tal como la clase de los seres humanos) que se distinguen por ciertas propiedades fácticas que están mencionadas en principios morales como condiciones para ser acreedor a ciertos derechos (p. 140).

Rechazar esta presuposición implica un cambio radical de estrategia filosófica que consistiría en lo siguiente:

1. Determinar primero cuáles son los principios morales de los que los derechos básicos derivan.

2. Definir luego las personas morales como la clase de todos aquellos individuos (o entidades) que poseen las propiedades que son fácticamente necesarias para gozar o ejercer tales derechos.

Aunque estos principios no son el foco de mi análisis, es necesario mencionarlos para completar el contexto de la argumentación. Los principios en cuestión son: el principio de inviolabilidad de la persona, el principio hedonista, el principio de autonomía y el principio de dignidad de la persona (pp. 141-145). Tales principios -de los que se derivan los derechos humanos- son categóricos pues "no condicionan el título a tales derechos a la posesión de una u otra característica" (p. 140). Son principios que se "aplican a todos o a todo" [everybody or everything] (p. 140). El segundo componente del cambio de estrategia presupone, según Nino, la distinción entre ser titular de un derecho y estar capacitado para ejercerlo. La idea es que "la personalidad moral es un concepto relacionado no con el hecho de ser titular de derechos morales fundamentales sino con el hecho de poseer las condiciones para ejercerlos o gozar de ellos" (p. 140). Esto significa que: "no hay garantía a priori de que todas las personas morales sean seres humanos, de que todos los seres humanos sean personas morales, y de que todos los seres humanos tengan el mismo grado de personalidad moral" (p. 140).

Según Nino, estas conclusiones

no parecen ahora objetables (como lo eran cuando se referían a la titularidad de los derechos) ya que no están basadas en una limitación 
arbitraria del alcance de nuestros principios morales, sino en hechos claros que determinan la posibilidad o imposibilidad de ejercer los derechos que derivan de aquellos (pp. 140-141).

\section{III}

Antes de elaborar, en la siguiente sección, un par de objeciones a la propuesta de Nino que requieren de la presentación de un elemento adicional a los ya expuestos, quisiera detenerme un momento a discutir su crítica a la interpretación analítica. Al hacerlo, creo que pueden salir a la luz algunas cuestiones de interés.

Me parece que no es correcto referirse a la interpretación analítica como imponiendo "limitaciones arbitrarias" al alcance de nuestros principios morales. Es posible considerar el criterio biológico del tipo "tener células con 46 cromosomas en su núcleo", como arbitrario porque es un criterio biológico, como parece hacerlo $\mathrm{Nino}^{6}$. El problema es que el criterio que se centra en la racionalidad, inteligencia, capacidad de elección y de elegir valores, que Nino prefiere por sobre el criterio de las células con 46 cromosomas, también debería ser entendido en términos (al menos en parte) biológicos, dadas las propiedades más primitivas de las que está compuesto (por ejemplo, el que alguien sea más o menos racional, puede explicarse, entre otras cosas, también por cuestiones de carácter biológico). Esto significa que, si es correcto afirmar que el criterio "células con 46 cromosomas" es moralmente arbitrario porque es biológico, habría que conceder entonces que las capacidades que Nino considera como moralmente relevantes también son arbitrarias en la medida en que algunas propiedades primitivas de las que están compuestas son biológicas. Por este motivo estas capacidades serían también propiedades fácticas, es decir, serían el tipo de propiedades que la estrategia tradicional usa para determinar el alcance de los principios morales fundamentales. Desde luego, hablar de "células con 46 cromosomas" es hablar de una propiedad biológica y hablar de, por ejemplo, "racionalidad" es hablar de una propiedad que tiene entre sus elementos constitutivos a propiedades biológicas, entre otras que no lo son, como el contexto social y familiar que,

6 "Pero debemos preguntarnos si tal concepto [el concepto de hombre definido en términos biológicos] puede también ser relevante a los propósitos de adscribir consecuencias normativas tan importantes como el ser acreedor a los derechos morales fundamentales. La respuesta difícilmente sea positiva, desde que es difícil ver cómo un hecho puramente biológico -como puede ser el tener células con 46 cromosomas en su núcleo- deba ser moralmente relevante" (p. 139). 
por lo demás, son tan arbitrarios moralmente como podría serlo un criterio puramente biológico. Sin embargo, si lo que hace que una propiedad sea moralmente arbitraria es que sea biológica, no se ve por qué la o las propiedades que puedan componer la capacidad "racionalidad" (o "capacidad para elegir", o etc.) no podrían contaminar la propiedad así compuesta al punto de volverla moralmente arbitraria. Es decir, si la propiedad "células con 46 cromosomas" es moralmente arbitraria porque es biológica, la propiedad "racionalidad" también sería moralmente arbitraria en la medida en que no parece razonable separar por completo la racionalidad de la biología. La diferencia estaría entonces, no entre una propiedad que es biológica y otra que no lo es, sino entre una propiedad que es moralmente relevante y otra que no lo es, donde la razón para dicha relevancia moral no debería ser el mero hecho de no ser una propiedad biológica. Comparto la idea de que determinar el contenido de una noción tan importante como la de persona moral -que Nino vincula con el concepto de "ciudadanía moral" (p. 140)- mediante una teoría biológica, como la que está en juego en el criterio de los 46 cromosomas, es ciertamente inadecuado. Si hablamos de ciudadanía moral, lo que debería estar en juego a la hora de determinar quiénes tienen tal ciudadanía es una teoría moral, pero aceptar eso no implica dejar fuera de consideración del razonamiento moral a posibles criterios biológicos. Lo que una teoría moral debería hacer es relacionar normativamente (de manera justificada, por cierto) propiedades biológicas de cierto tipo -aunque no solo propiedades biológicas- con principios o consideraciones morales que prescriban ciertos tipos de trato, entre los que se cuenta la distribución de bienes estimados relevantes, como lo son los derechos fundamentales. Piénsese, por ejemplo, en ese criterio comúnmente aceptado que hace de la capacidad de sentir placer y dolor una propiedad que demanda, desde el punto de vista moral, cierto tipo de consideración. ${ }^{7}$

El problema con el criterio que se centra en la racionalidad, inteligencia, capacidad de elección y de elegir valores no sería su irrelevancia moral sino su gradualidad, es decir, el hecho de que las capacidades descritas son poseídas por los seres humanos en distintos grados. Lo que resulta problemático es que, del uso del criterio de la racionalidad, se sigue la extensión desigual de los derechos morales. Pero este es otro problema, uno que no tiene que ver con la arbitrariedad de una limitación sino con

${ }^{7}$ En Singer (1989, cap. 1) se encuentra un argumento que, apoyándose en esta capacidad, amplía el concepto de igualdad moral a animales y seres humanos. Si bien no me parece que su argumentación sea convincente, no es esta la ocasión para analizar el punto. 
la gradualidad en la posesión de las capacidades en virtud de las cuales los seres humanos poseen ciertos derechos. El punto-si seguimos la estrategia tradicional-es que los seres humanos no son iguales porque no poseen las propiedades relevantes en el mismo grado y, en consecuencia, en virtud del principio de superveniencia citado más arriba, no deberían tener iguales derechos morales. Esto mostraría que, usando propiedades naturales, no disponemos de buenas razones para basar la igual atribución (titularidad) de derechos morales en la creencia de que los seres humanos son iguales y de que, por tanto, quienes son personas morales (en la terminología de Nino), son personas morales iguales.

Paso ahora a exponer brevemente un elemento que completa el argumento de Nino a favor del cambio radical de estrategia. Como hemos visto, lo que Nino propone es basar el concepto de persona moral en la posesión de ciertas capacidades, no con el fin de fundar en ellas la titularidad de los derechos fundamentales -como lo hace la estrategia tradicional-, sino con el fin de justificar el ejercicio de tales derechos. Estas capacidades serían las que determinan la aplicación de los cuatro principios morales arriba mencionados y serían las siguientes (pp. 145-146):

1. En relación al principio de autonomía: elegir y materializar principios morales generales e ideales de bondad personal y llevar a cabo planes de vida que sean referencia específica de ese principio.

2. En relación al principio hedonista: sentir dolor y placer.

3. En relación al principio de la inviolabilidad de la persona: ser consciente de que se es independiente y centro de interés irreemplazable.

4. En relación al principio de la dignidad de la persona: ser capaces de tomar decisiones y consentir las consecuencias de sus actos.

Desde el punto de vista ideal, una persona moral completa es alguien que posee estas cuatro capacidades en su alcance máximo (p. 146). Hay dos dimensiones en las que la personalidad moral puede variar, según Nino:

a. Variaciones de grado en las capacidades.

b. Variaciones en la presencia de ciertas capacidades.

En ambas dimensiones de variación, una cualitativa y la otra cuantitativa, un ser puede ser más o menos persona moral. Si, por ejemplo, el ser humano A y el ser humano B tienen las mismas capacidades pero A las tiene en mayor grado que B, A es más persona moral que B. Si el ser humano $\mathrm{C}$ tiene menos capacidades que el ser humano $\mathrm{A}, \mathrm{C}$ es menos persona moral que A. Dejo de lado aquí la discusión relativa a las variaciones de grado o de número que se deban a cuestiones de potencialidad 
y que nos podrían llevar a considerar a un determinado ser como menos persona moral que otro, sin que de eso se desprendan necesariamente consecuencias normativas desiguales para quien es, en este momento del tiempo, menos persona moral, o que nos podrían llevar a considerar a un ser que tiene menos capacidades relevantes en este momento del tiempo como siendo igual persona moral que un ser que tiene más.

¿Para qué nos serviría todo esto? ¿Qué es lo que el cambio radical de estrategia filosófica de Nino mejora respecto de la estrategia tradicional? Por un lado, evitar condicionar la extensión de derechos morales (es decir, su titularidad) a ciertas propiedades fácticas. Como hemos visto, cuando definimos el concepto de persona moral usando la estrategia tradicional, si no existe ninguna propiedad natural que todos posean en igual grado, la extensión de derechos se ve afectada (p. 140). Para disolver este problema Nino propone, primero, considerar los principios morales de los que derivan los derechos morales como aplicables a todos o a todo y, segundo, distinguir entre la titularidad y el ejercicio de los derechos para afirmar luego que el goce o ejercicio de estos derechos depende de si se poseen las capacidades arriba mencionadas y en qué grado. No queda claro si lo que lleva a Nino a rechazar la estrategia tradicional es que esta justifica la desigual titularidad de derechos fundamentales, o si el problema es que dicha estrategia usa criterios arbitrarios (independientemente de si tales criterios llevan a una igual o desigual titularidad). Es claro que ambas interpretaciones no son compatibles pues, si el problema es que mediante esta estrategia se justifica una desigual titularidad de derechos, no es posible objetar también que dicha desigual titularidad se basa en criterios arbitrarios. Pero si el problema es la arbitrariedad, esta parece reducirse al uso de propiedades biológicas que, por el hecho de ser biológicas, son arbitrarias. Hemos visto que hay algunos problemas con esto. Por otra parte, cuando la propiedad no es biológica y es moralmente relevante, lo que resultaría arbitrario sería establecer una igual titularidad de derechos fundamentales y no una desigual titularidad, que es lo que debería seguirse cuando se usan criterios no biológicos moralmente relevantes que no todos tienen en igual grado.

IV

Creo que hay, además del punto recién mencionado, al menos dos problemas con la propuesta de Nino. Primero, no se entiende la cuestión de los principios morales fundamentales como principios que se aplican a todos o a todo. Con esta idea seguramente se gana en la extensión de 
derechos -la titularidad de estos ya no depende de propiedades fácticas desigualmente poseídas- pero al precio de una dosis de oscuridad metafísica poco deseable o, si debemos interpretar la cuestión literalmente, al precio de considerar las cosas (las piedras y plantas, por ejemplo) como teniendo derechos. Nino afirma: "No es que, por ejemplo, una piedra no tenga el derecho a la libertad de movimiento; este derecho es insignificante para la piedra desde que carece de la capacidad de decidir un plan de vida para el cual la libertad de movimiento constituye un prerrequisito" (p. 145). Deberíamos concluir, entonces, que las piedras tienen derecho a la libertad de movimiento pero que tal derecho es insignificante para ellas. ¿De qué sirve afirmar esto? A Nino le sirve para evitar basar la adscripción de derechos en propiedades fácticas (biológicas o no, si tal distinción es posible). Negarles a las piedras la titularidad de derechos porque son piedras implicaría hacer justamente eso. ${ }^{8}$ El punto problemático es que Nino pretende resolver el problema de la desigual titularidad de derechos a la que nos llevaría la estrategia tradicional simplemente decretando que los principios morales de los que se derivan tales derechos se "aplican a todo y a todos" (p. 140). La conclusión que se sigue es bastante evidente: puestas así las cosas, una desigualdad en la titularidad de derechos es algo que sencillamente no se puede dar. En ausencia de un argumento que justifique considerar los principios morales de esta forma, la conclusión a la que se llega no tiene mayor sustento.

Segundo, respecto de la variación de la persona moral (excluyendo, como decía, cuestiones de potencialidad), las cosas podrían ponerse del siguiente modo. Es persona moral "completa" (p. 146) quien tiene las cuatro capacidades mencionadas. Esta sería "una condición ideal” (p. 146). El punto que no está del todo claro aquí es cómo es que, en relación con estas capacidades, la condición de persona moral varía. El que sea una condición ideal isignifica que, (a) quién carezca de una de estas capacidades sigue siendo una persona moral igual a quien es una persona moral completa, o significa que (b) quién carezca de una de estas capacidades es persona moral pero no igual a quien es una persona moral completa, o significa que (c) quién carezca de una de estas capacidades

${ }^{8}$ En el caso de la piedra el punto podría parecer poco importante dada la completa ausencia de intereses que debe atribuírsele. Pero, en otros casos, las consecuencias podrían ser bastante menos inocuas normativamente. Piénsese, por ejemplo, en el derecho a la vida que tendrían los ratones, derecho cuyo ejercicio, en virtud de la capacidad para sentir placer y dolor que estos tienen, habría que cautelar. Agradezco a un referí anónimo de esta revista haberme señalado el punto. 
no es una persona moral pero sí tiene derecho a que esa única capacidad, (¿o esas dos o tres capacidades?), sean protegidas? En los casos (a) y (b) la variación cuantitativa no afectaría la condición de ser una persona moral, aunque (b) admite que, quien tiene menos capacidades, es menos persona moral que quien tiene más. En (c) la variación cuantitativa sí afectaría dicha condición sin que ello implique pérdida de estatus moral; es decir, a pesar de tener solo una capacidad, el valor moral de esta justifica su protección. Parece ser que esta es la posición de Nino, por lo menos cuando la única capacidad poseída es la de tener sensaciones placenteras o dolorosas (p. 146). Cuando este no es el caso y el ser en cuestión tiene todas las capacidades menos la capacidad de tener sensaciones placenteras o dolorosas, parece razonable considerar a ese ser como una persona moral. ${ }^{9}$ Bajo esta alternativa, si un ser no tiene las 4 capacidades, entonces no es una persona moral solo si la capacidad de la que carece no es la de tener sensaciones placenteras o dolorosas. ¿Cuál de estas capacidades? ${ }^{10}$ Pero, se podría negar que una variación en la dimensión cuantitativa implique no ser una persona moral. Podría sostenerse que, si un ser carece solo de una de estas propiedades, continúa siendo una persona moral. El problema evidente con esta posibilidad es que, a falta de un argumento, el límite numérico que se establezca como criterio para considerar a un ser como teniendo personalidad moral parece inevitablemente arbitrario. A esto se agrega el que, dada la conexión profunda que parece razonable suponer entre algunas de las capacidades mencionadas por Nino, quizás la única capacidad de la que un ser pueda carecer sin tener que carecer también de alguna otra, sea la de tener sensaciones placenteras o dolorosas. Sin embargo, podría sostenerse que, basta que una de estas capacidades esté presente para que, quien la posee, sea considerado una persona moral. Esto implicaría entender la personalidad moral como una propiedad binaria pues, si se tiene una

${ }^{9}$ Lo razonable de esta consideración descansa en la profunda conexión que hay entre las capacidades 1, 3 y 4 mencionadas en la sección III. Recordemos que estas capacidades son: (1) elegir y materializar principios morales generales e ideales de bondad personal y llevar a cabo planes de vida que sean autónomos; (2) sentir dolor y placer; (3) ser consciente de que se es independiente y centro de interés irreemplazable; (4) ser capaz de tomar decisiones y consentir las consecuencias de sus actos. La profundidad de la conexión se explica así: parece poco plausible suponer que alguna de estas capacidades podría existir sin la otra (o, al menos, la conexión es especialmente profunda entre las capacidades 1 y 4 ).

10 Atendiendo a lo dicho en la nota precedente, podría sostenerse que, dada la conexión profunda entre las capacidades en cuestión, un escenario en el que se tenga la capacidad 1 pero no la 4 no parece plausible. 
capacidad o tres (de las cuatro mencionadas por Nino), la personalidad moral no varía. Tal condición solo puede desaparecer y, además, hacerlo en caso de que el ser en cuestión no tenga ninguna de estas capacidades. Esto implicaría también considerar la capacidad para tener sensaciones placenteras o dolorosas como suficiente para ser persona moral, cuestión ciertamente discutible y que, además, Nino no afirma.

Entender la personalidad moral como una propiedad binaria acercaría (polémicamente) la posición de Nino a la de John Rawls, así como esta es expuesta en el parágrafo 77 de su Teoría de la justicia (1971), titulado "Las bases de la igualdad". Rawls sería un representante de la estrategia tradicional y alguien que Nino extrañamente no toma en consideración. Según Rawls la igualdad necesita de bases naturales con el fin de identificar la clase de entidades [creatures, p. 444] a las que se aplica la justicia igual. La idea es que una propiedad moralmente relevante puede ser predicada de aquellos seres que tienen ciertas propiedades escalares dentro de un cierto rango. Este tipo de propiedad es llamada "propiedad de rango" (o propiedad binaria): es la propiedad de poseer ciertas propiedades escalares dentro de un cierto rango. Para Rawls, la propiedad de rango relevante es la "personalidad moral", que existe en virtud de la posesión, por parte de los individuos, de un mínimo de ciertas capacidades básicas de agencia, como lo son el sentido de la justicia y el sentido del propio bien. Rawls ofrece aquí el siguiente ejemplo: la propiedad de estar al interior del círculo es una propiedad de rango de los puntos en el plano. "Todos los puntos al interior del círculo tienen esta propiedad aunque sus coordenadas varían en un cierto rango. Y ellos tienen igualmente esta propiedad, ya que ningún punto interior a un círculo es más o menos interior que cualquier otro punto" (p. 446). Esto significa que, descriptivamente hablando, dada la existencia de la propiedad de rango "personalidad moral", disponemos de una propiedad fáctica para justificar la igual distribución de derechos entre quienes la poseen, sin tener que concentrarnos en los grados de las propiedades escalares que componen tal propiedad. No voy a examinar en detalle las razones que se pueden ofrecer para no concentrarnos en las propiedades escalares (eso ciertamente es un punto problemático ${ }^{11}$ ). Aunque Rawls no es explícito sobre este punto, es plausible sostener que, dada su posición respecto de la relación entre capacidades y lotería natural, concentrarse en las propiedades escalares y derivar de allí ciertas consecuencias normativas sería moralmente arbitrario. Esto muestra

11 Una justificación para no concentrarse en las propiedades escalares se encuentra en Carter (2011). 
-muy esquemáticamente- que existe la posibilidad de ofrecer una justificación descriptiva o fáctica de la igualdad básica que no implica negar que las propiedades seleccionadas varíen en grado, sin que ello nos lleve a justificar la desigual posesión de, por ejemplo, derechos fundamentales.

Pareciera que lo que Nino ha logrado con su cambio de estrategia es simplemente desplazar el problema de la variación en los grados de las propiedades relevantes desde la titularidad de los derechos al ejercicio de ellos. ¿En qué sentido lo segundo sería mejor que lo primero? Si lo primero llevaba a conclusiones elitistas ¿por qué lo segundo no? Lo que hace que un ser pueda gozar de tales y cuales derechos es la posesión de tales y cuales características. Si un ser A tiene la capacidad x gozará del derecho x', pero si un ser B tiene la capacidad x en menor grado que A, deberíamos concluir que debería gozar del derecho x' en menor grado. Es decir, a variaciones en la personalidad moral deberían corresponder variaciones en el ejercicio de los derechos. Esto suena tan elitista como afirmar que algunos seres humanos tienen ciertos derechos que otros no tienen, simplemente porque estos últimos tienen las capacidades relevantes en menor grado. Si la respuesta a esto es que basta que la capacidad x esté, sin importar su grado, para que todos quienes la poseen gocen del derecho x' sin distinciones de grado, sería necesario dar un argumento semejante al que habría que ofrecer para justificar el tratamiento que hace Rawls de la personalidad moral como una propiedad de rango. Pero, independientemente de esto, ¿qué hay de ventajoso, desde el punto de vista moral, en evitar la desigual titularidad de derechos que, según Nino, se deriva de la estrategia tradicional, para pasar luego a elaborar un argumento que legitima el desigual ejercicio de los derechos morales según la desigualdad en las capacidades? Si lo primero parece moralmente objetable ¿por qué lo segundo no?

\section{Bibliografía}

Arneson, R. (1999), "What, If Anything, Renders All Humans Morally Equal?", en Jamieson, D. (ed.), Singer and His Critics, Oxford, Blackwell.

Carter, I. (2011), "Respect and the Basis of Equality", Ethics, 121, pp. 538-571.

Carter, I. y Page, O. (ms.), "The Basis and Value of Distributive Equality". Cupit, G. (2000), "The Basis of Equality", Philosophy, 75, pp. 105-125.

Lloyd Thomas, D. A. (1979), "Equality Within the Limits of Reason Alone", Mind, 88, pp. 538-553. 
Nino, C. S. (1987), "The Concept of Moral Person", Crítica, XIX(56), pp. 47-75.

(2007), "El concepto de persona moral", en Maurino, G. (ed.), Derecho, moral y política II, Barcelona, Gedisa.

Rawls, J. (1971), Teoría de la justicia, México, FCE.

Singer, P. (1989), Liberación animal, Madrid, Trotta.

Waldron, J. (2002), God, Locke, and Equality, Cambridge, Cambridge University Press.

_- (2008), "Basic Equality", New York School of Law, Public and Legal Theory Research Paper Series, $\mathrm{N}^{\circ}$ 08-61.

Williams, B. (1962), "The Idea of Equality", en Laslett, P. y Runciman, W. G. (eds.), Philosophy, Politics and Society, Series II, Oxford, Blackwell.

Recibido el 15 de diciembre de 2014; aceptado el 12 de febrero de 2015. 